\title{
Hyperurikåmie
}

\section{Neue Möglichkeiten zur Diagnosesicherung und Therapiekontrolle}

Goldstandard zur Sicherung der Gicht sei lange Zeit die Gelenkpunktion mit anschließender Visualisierung der Kristalle im Polarisationsmikroskop gewesen, berichtete Prof. Wolfgang

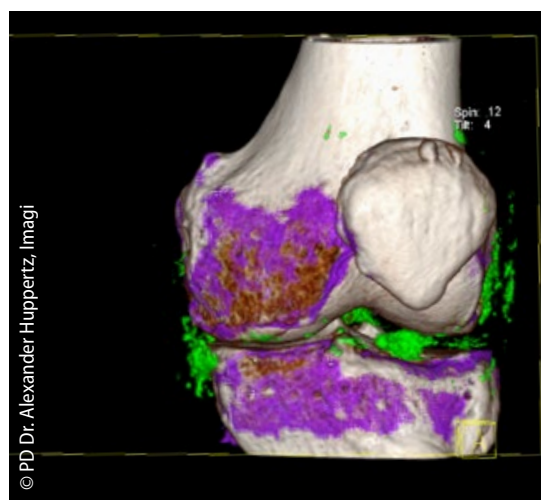

DECT-Aufnahme des rechten Knies mit multiplen Uratablagerungen.
Schmidt, Berlin. Die Methode werde jedoch von Allgemeinmedizinern nur selten angewendet, vorrangig wegen der nötigen Punktion und der fehlenden Erfahrung von Hausärzten. Deutlich besser geeignet sei hier die Arthrosonografie, bei der Harnsäureablagerungen durch das typische Doppelkonturzeichen am Gelenkspalt nachweisbar sind.

Modernstes Instrument zum Nachweis von Harnsäureablagerungen ist gegenwärtig die Dual-Energy-Computertomografie (DECT). Sie arbeitet mit zwei um $90^{\circ}$ versetzten rotierenden Elektronenquellen unterschiedlicher Energie (80 bzw. $140 \mathrm{kV}$ ). Die beiden hierbei erzeugten Bilder werden durch eine spezielle Software miteinander verglichen, die dann die Natriumurat-Ablagerungen farblich hervorhebt.
Bei Patienten, bei denen die Diagnose symptomatische Hyperurikämie gestellt wird, sollte wie in den Leitlinien der EULAR empfohlen, eine Therapie mit dem Zielwert von $<6 \mathrm{mg} / \mathrm{dl}(360 \mu \mathrm{mol} / \mathrm{l})$, in schweren Fällen auf $<5 \mathrm{mg} / \mathrm{dl}$, erfolgen. Hierbei erwies sich der nicht-selektive Xanthinoxidasehemmer Febuxostat hinsichtlich der Harnsäuresenkung als effektiver als Allopurinol. Er kann auch bei Patienten mit leichter bis mittelschwerer Nierenfunktionseinschränkung ohne Dosisanpassung eingesetzt werden. $\mathrm{Zu}$ dem ist Febuxostat Allopurinol im Hinblick auf die Reduktion von oxidativem Stress und Entzündungen überlegen und besitzt einen positiven Einfluss auf Nieren und Gefäße.

Red.

\section{Aut idem bei Inhalationsgeräten}

\section{Hausärzte sollten das ausschließen}

_ Die bei Asthma und COPD gebräuchlichen Inhalationsgeräte sollten vom Apotheker nicht ausgetauscht werden, betonen Bundesverband der Pneumologen (BdP) sowie Deutscher Allergie- und Asthmabund (DAAB). Sie raten Ärzten, bei der Verordnung von inhalativen Atemwegsmedikamenten Aut-idem auszuschließen.

Die Politik drängte darauf, die Therapie bezahlbar zu halten, indem sie für alle Devices nur eine Festbetragsgruppe definiert. Nun wurde zudem die AutIdem-Regelung gesetzlich beschlossen: Der Apotheker kann künftig wirkstoffgleiche Therapien durch kostengünstigere Alternativen ersetzen.

Lungenfachärzte wie der stellvertretende Bundesvorsitzende des BdP Dr. Thomas Hering, Berlin, sehen dadurch ihre therapeutischen Bemühungen untergraben. „Es gibt kleine Unterschiede bei den Substanzen, aber große Differenzen bei den Devices“, so Hering. Für den Therapieerfolg ist die Wirkstoffdeposition essenziell, aber gut die Hälfte aller Patienten machen bei der Anwendung Fehler.

„Wir empfehlen Hausärzten, sich die Geräte genau anzusehen: Kommt der Patient mit der Anwendung zurecht? Kann er die Dosis variieren, Mono- und Kombinationstherapien aus dem gleichen Gerät bedienen?" Laut Hering müssen Patienten konsequent geschult und die korrekte Handhabung überprüft werden. Dann aber sollte der Patient auch bei seinem Device bleiben.

Inhalationsgeräte gehören deshalb auf die sog. Substitutionsausschlussliste, fordert Hering. Solange dies nicht der Fall ist, sollten Ärzte Aut-idem ausschließen. Einer Befragung unter 300 Lungenfachärzten zufolge tun dies in der Regel 75\% der Pneumologen. Bei Hausärzten geschieht dies nach Einschätzung der Fachärzte aber nur selten.

\section{Dr. Dirk Einecke}

- Pressekonferenz Unterschätzte Volkskrankheit - Patienten und Ärzte sehen erhebliches Verbesserungspotential in der Versorgung von Patienten mit Erkrankungen der Atemwege in Deutschland", Berlin, Mai 2016 (Veranstalter: GSK)

\section{Kurz notiert}

Spannungskopfschmerz $\rightarrow$ Auf dem diesjährigen Kongress der Gesellschaft für Phytotherapie wurde eine apothekengestützte nicht-interventionelle Studie präsentiert, in der die Wirksamkeit der Behandlung von Kopfschmerzen vom Spannungstyp mit einer 10\%-igen Pfefferminzöllösung (Prüfpräparat: Euminz ${ }^{\circledast}$ ) erneut bestätigt wurde: Mehr als 80\% der 271 Studienteilnehmer beurteilten die Wirksamkeit sowie über 97\% die Verträglichkeit des Prüfpräparates als sehr gut und gut. Bereits am ersten Tag verschwanden die Kopfschmerzen bei $43 \%$ der Studienteilnehmer komplett. Bei weiteren $44 \%$ wurden die Beschwerden deutlich reduziert. Das Fazit von Dr. Ronald Brand, Königstein: „Die Schmerzlinderung bei leichten bis mittleren Kopfschmerzen und die Dauer bis zum Wirkungseintritt ist mit herkömmlichen, systemischen Schmerzmitteln vergleichbar - nach 1530 Minuten ist eine Linderung der Schmerzen zu verspüren“. Euminz ${ }^{\circledR}$ ist in Deutschland als einziges lokal anzuwendendes natürliches Arzneimittel zur Behandlung von leichten bis mittelschweren Spannungskopfschmerzen zugelassen. Auch für Kinder ist das Pfefferminzöl verordnungsund erstattungsfähig.

Red.

- Nach Informationen von MCM 